\title{
BEM-numerics and KdV-model analysis for solitary wave split-up
}

\author{
E. F. G. van Daalen, E. van Groesen, S. R. Pudjaprasetya
}

Abstract In this paper we consider travelling surface waves on a layer of water of decreasing depth. A numerical scheme based on the boundary element method is used to present calculations for the run-up of a solitary wave. The numerical results are compared with an analytical approximation based on a modified Korteweg-de Vries equation.

\section{1}

\section{Introduction}

In this paper we consider some aspects of travelling surface waves on a layer of water (considered as an ideal fluid) of decreasing depth. The very accurate numerical scheme based on the Boundary Element Method (BEM), as developed by van Daalen $(1993,1995)$, is used for two different aims:

The first aim is to present BEM-calculations for the runup of a solitary wave. The results will show that (for limited changes in depth) the solitary wave splits into two clearly distinguishable waves. Such a splitting may be thought to be well-known in view of the vast amount of literature about this subject that is available since the seventies, see e.g. Grimshaw (1970, 1971), Johnson (1972, 1973, 1994), Karpman and Maslow (1978), Knickerbocker and Newell (1980), and Newell (1985). However, all these references use some model equation, mostly a variant of

Communicated by T. A. Cruse, 2 August 1996

E. F. G. van Daalen

University of Twente, Department of Mechanical Engineering, P.O. Box 217, 7500 AE Enschede, The Netherlands

E. van Groesen

University of Twente, Department of Applied Mathematics, P.O. Box 217, 7500 AE Enschede, The Netherlands

S. R. Pudjaprasetya

Institut Teknologi Bandung, Department of Mathematics, Jalan Ganesha 10, Bandung 40132, Indonesia

Correspondence to: E. F. G. van Daalen

These investigations were done while the first author was working at the Department of Applied Mathematics of the University of Twente, with financial support from the Royal Netherlands Academy of Arts and Sciences (KNAW). Support from the National Foundation for Supercomputing Facilities (NCF) is gratefully acknowledged. The contribution of the second author was financed by part of the Joint Research Project CI1*-CT93-0018 between UT-Math and ITB-Math. the KdV-equation, with coefficients or perturbations that should take the changing depth into account. The BEMcalculation, in contrast, is based on the exact equations, that is without using some approximation like the Boussinesq conditions on the wave shape, and without a priori assumptions such as uni-directional wave propagation.

The second aim is to compare the numerical results (considered to be the 'exact' description of the phenomenon) with a relatively simple analytical description. This analytical work uses a specific modification of the KdVequation, called ' $\mathrm{KdV}$-top', that was derived by van Groesen and Pudjaprasetya (1993) under the assumption of mild bottom variations and waves that satisfy Boussinesq conditions. By comparing the model results with those of the BEM-calculations, we are able to investigate the validity of the model description. Usually a comparison between the exact equations and an approximate model cannot be made in detail. In this case we do have the opportunity to make such a comparison; for instance, we can estimate a parameter $\epsilon$, that enters from the Boussinesq assumption, in such a way that the $\mathrm{KdV}$-top model provides the best possible results (over a long range of wave forms).

The organization of this paper is as follows: in Section 2 we present the full set of equations, which is used in the BEM-calculations, and the approximate model equation KdV-top, with the underlying assumptions for the validity of the model. In Section 3 we consider the specific case of an even bottom (constant depth), and investigate and compare the solitary waves of both models. In Section 4 we describe the numerical aspects of the BEM-calculations, both the principles of the algorithm and a discussion of the computer performance in the specific calculations of the solitary wave splitting. In Section 5 an approximate analytic description is presented of the splitting process within the KdV-top model. It is shown that conservation of mass and energy alone suffice to obtain the quasi-homogeneous approximation. In Section 6 we present the results of the BEM-calculations and the quasi-homogeneous description of the splitting and compare the results. Conclusions are presented in Section 7.

\section{2}

Exact equations and model equations

In this section we present the full set of equations that will be used in the BEM-calculations, and the approximate model equation KdV-top, with the underlying assumptions for the validity of the model. 
2.1

Boundary value problem for the exact equations

For an ideal fluid (as water is in a good approximation), extending in the horizontal $x$-direction above a bottom described by the vertical coordinate $z=-h(x)$, and considering irrotational flow, the full set of equations is described in terms of a surface elevation $\eta(x, t)$ and a velocity potential $\Phi(x, z ; t)$ in the fluid domain

$\Omega(\eta)=\{(x, z) \mid-h(x)<z<\eta(x, t)\}$ by the following boundary value problem (BVP):

$\Delta \Phi \equiv \nabla^{2} \Phi=0 \quad$ in $\quad \Omega(\eta)$,

that is Laplace's equation for $\Phi$, with the zero-flux condition on the bottom boundary - see also Fig. 1:

$\frac{\partial \Phi}{\partial n} \equiv \nabla \Phi \cdot \vec{n}=0 \quad$ on $\quad z=-h(x)$.

The time dependent boundary conditions on the free surface are the kinematic condition and the dynamic condition (the Bernoulli equation)

$$
\begin{array}{ccc}
\eta_{t}=(\nabla \Phi \cdot \vec{n}) \sqrt{1+\eta_{x}^{2}} & \text { at } \quad z=\eta(x, t), \\
\Phi_{t}=-\frac{1}{2}|\nabla \Phi|^{2}-g z & \text { at } \quad z=\eta(x, t),
\end{array}
$$

where subscripts denote partial differentiation. In (4), $g$ is the gravitational acceleration acting in downward (negative $z$-) direction. Equations (3-4) are the essential sources of difficulties in this description.

In view of the following it is interesting and worthwhile to recall, see van Groesen and De Jager (1994), that

Eqs. (3-4) describe a Hamiltonian system; introduce the free surface potential $\varphi(x, t) \equiv \Phi(x, z=n(x, t), t)$, and consider the total energy (kinetic energy plus potential energy) as the Hamiltonian with canonical variables $\varphi$ and $\eta$ :

$H_{E}(\varphi, \eta) \equiv P(\eta)+K_{E}(\varphi, \eta)$,

where $P(\eta)$ and $K_{E}(\varphi, \eta)$ are the potential and kinetic energy respectively (the subscript $E$ indicating that the exact energy is considered). Neglecting surface tension, the potential energy is given by

$P(\eta)=\iint_{0}^{\eta} g z \mathrm{~d} z \mathrm{~d} x=\int \frac{1}{2} g \eta^{2} \mathrm{~d} x$

and the kinetic energy is

$K_{E}(\varphi, \eta)=\iint_{\Omega(\eta)} \frac{1}{2}|\nabla \Phi|^{2} \mathrm{~d} x \mathrm{~d} z$

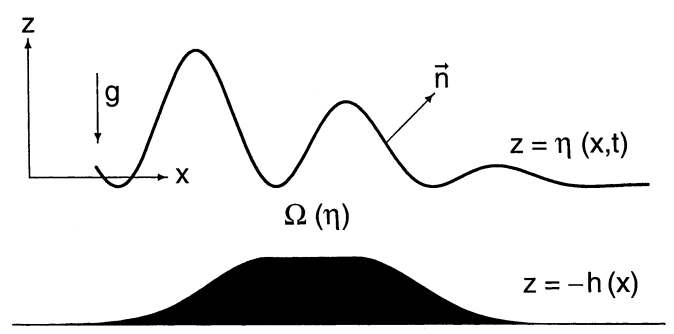

Fig. 1. Definition of fluid domain with bottom topography and free surface where $\Phi$ is the solution of the BVP (1-2) with

$\Phi(x, z=\eta(x)) \equiv \varphi(x)$. Then, Eqs. (3-4) can be written in the Hamiltonian form

$\partial_{t}\left(\begin{array}{l}\varphi \\ \eta\end{array}\right)=\left(\begin{array}{rr}0 & -1 \\ 1 & 0\end{array}\right)\left(\begin{array}{l}\delta_{\varphi} H_{E}(\varphi, \eta) \\ \delta_{\eta} H_{E}(\varphi, \eta)\end{array}\right)$

where $\delta_{\varphi}$ and $\delta_{\eta}$ denote the variational derivatives with respect to $\varphi$ and $\eta$ respectively.

However, instead of $\varphi$ it is more convenient to work with $u=\varphi_{x}$ and the Hamiltonian system is transformed to:

$\partial_{t}\left(\begin{array}{l}u \\ \eta\end{array}\right)=\left(\begin{array}{cc}0 & -\partial_{x} \\ -\partial_{x} & 0\end{array}\right)\left(\begin{array}{l}\delta_{u} H_{E}(u, \eta) \\ \delta_{\eta} H_{E}(u, \eta)\end{array}\right)$,

These equations have as conserved quantities the mass $\int \eta \mathrm{d} x, \int u \mathrm{~d} x$ and the total energy, which is precisely the Hamiltonian $H_{E}$. The horizontal momentum, given by

$$
\begin{aligned}
I_{E} & =\iint_{-h(x)}^{\eta(x, t)} \Phi_{x} \mathrm{~d} z \mathrm{~d} x \\
& =\int\left[\frac{\mathrm{d}}{\mathrm{d} x} \int_{-h(x)}^{\eta(x, t)} \Phi \mathrm{d} z-\left.\eta_{x} \Phi\right|_{z=\eta(x)}+\left.h_{x} \Phi\right|_{z=-h(x)}\right] \mathrm{d} x \\
& =\int u \eta \mathrm{d} x-\int h^{\prime}(x) \Phi(x,-h(x)) \mathrm{d} x
\end{aligned}
$$

is not conserved, because the translation symmetry is lost, except when the bottom is flat.

\section{2}

KdV-top(ography) model

A simpler set of equations can be obtained by restricting to a specific set of wave forms. The usual Boussinesq-type of waves are waves that are 'rather' low and 'rather' long, more specifically described by waves with amplitude of order $\epsilon$, and wavelength of order $\epsilon^{-1 / 2}$. For such waves the effect of nonlinearity and lowest order dispersion are of comparable order. When the variations in the bottom topography extend over even larger distances, specifically of order $\epsilon^{-2}$, the equations, correct in order $\epsilon^{2}$, are of Boussinesq-type. They can easily be found by approximating the Hamiltonian (the kinetic energy part) as an explicit approximate expression in the canonical variables. The set of equations retains the Hamiltonian structure as in (9), with $H_{E}$ replaced by the approximated Hamiltonian

$H_{B}(u, \eta)=\int\left[\frac{1}{2} g \eta^{2}+\frac{1}{2} h(x) u^{2}+\epsilon\left(-\frac{1}{6} h^{3}(x) u_{x}^{2}+\frac{1}{2} \eta u^{2}\right)\right] \mathrm{d} x$.

A further simplification can be obtained by restricting to uni-directional waves, thereby reducing the set of two equations of first order in time to a single equation of first order in time. This uni-directionalization can also be invoked by further simplifying the Hamiltonian $H_{B}$. In fact, in this process, the two canonical variables are related in the following way:

$u=\sqrt{\frac{g}{h}} \eta+\mathcal{O}(\epsilon)$

where the $\mathcal{O}(\epsilon)$-term comes from the reflected wave, see van Groesen and Pudjaprasetya (1993). Then the resulting 
equation can be expressed in the wave elevation only. The equation has retained a Hamiltonian structure and it is called $\mathrm{KdV}$-top(ography), given by,

$\partial_{t} \eta=-\Gamma(x) \delta_{\eta} H(\eta)+\mathcal{O}\left(\epsilon^{2}\right)$,

where $\Gamma(x)$ is the skew-symmetric operator

$\Gamma(x)=\frac{1}{2}\left[c(x) \partial_{x}+\partial_{x} c(x)\right] \quad$ with $\quad c(x)=\sqrt{g h(x)}$,

and $H$ is a further approximation of the total energy:

$H(\eta)=\int\left[\frac{1}{2} \eta^{2}+\epsilon\left(-\frac{1}{12} h^{2}(x) \eta_{x}^{2}+\frac{\eta^{3}}{4 h(x)}\right)\right] \mathrm{d} x$.

Explicitly, the KdV-top equation reads:

$\partial_{t} \eta=-\Gamma(x)\left[\eta+\epsilon\left(\partial_{x}\left(\frac{1}{6} h^{2}(x) \eta_{x}\right)+\frac{3 \eta^{2}}{4 h(x)}\right)\right]+\mathcal{O}\left(\epsilon^{2}\right)$.

It should be observed that when $h(x)$ is constant, this is just the standard $\mathrm{KdV}$-equation (in physical coordinates). The Hamiltonian $H$ in (15) is the approximate total energy times $1 / 2 g$; the linearized equation of (16) for a flat bottom $h_{0}$ has travelling wave solutions with linear velocity $c_{0}=\sqrt{g h_{0}}$.

The details of the derivation above can be found in van Groesen and Pudjaprasetya (1993), where it is also shown that Eq. (16) is equivalent to the equations derived by Johnson (1973) and Newell (1985); the present description is given in more physical terms.

For the rest of this paper it is important to observe that the KdV-top equation has in general the same two conserved quantities as the full set of equations. (For constant depth $h$, the $\mathrm{KdV}$-equation is completely integrable and has infinitely many conserved quantities.) These are now expressed as the generalized mass

$M(\eta)=\int \frac{\eta}{\sqrt{c(x)}} \mathrm{d} x$

and the approximate energy, which is the Hamiltonian $H$ of (15) itself.

Write the first term in the horizontal momentum (10), using (12), in $\eta$ only and multiply by $1 / 2 g$ (just like for $H$ ), then we have the following generalized momentum:

$I(\eta)=\int \frac{\eta^{2}}{2 c(x)} \mathrm{d} x$

which is not conserved since translation symmetry is lost (except when $h$ is constant).

A final remark about the effect of bottom reflections: the $\mathrm{KdV}$-top equation neglects the effects of reflection of a uni-directional wave completely. In the assumption of very mild bottom variations this is justified. But for somewhat steeper bottom variations, the lowest order effect of the reflection can be taken care of by introducing a forcing term in the equation; the result is then - see van Groesen and Pudjaprasetya (1993):

$\partial_{t} \eta=-\Gamma(x) \delta_{\eta} H(\eta)-\mathscr{F}+\mathcal{O}\left(\epsilon^{2}\right)$, with a forcing $\mathscr{F}$ that depends on bottom variations and the initial elevation; it is of the order $\epsilon^{\mu}$, with $1<\mu<2$.

3

\section{Comparison of solitary waves}

In this section we consider the specific case of an even bottom (constant depth) and investigate the solitary waves of both models. For a given depth and 'position' (dividing out an arbitrary translation), a one-parameter family of such wave exists. The momentum $\gamma$ of the waves will be used as the parameter, since it is a more stable quantity than the amplitude $a$, although there is a one-to-one correspondence. These solitary waves will be denoted by $S_{e}$ for the 'exact' set of equations, and by $S_{a}$ for the approximate $\mathrm{KdV}$-model.

\section{1}

Calculations of exact solitary waves $S_{e}$

A two-dimensional steady periodic solution of the nonlinear water wave problem with a flat bottom was proposed in the form of Fourier series for the stream function and the elevation by Rienecker and Fenton (1981). We implemented their approach in our numerical method, such that the corresponding series for the potential and the elevation read:

$$
\begin{aligned}
\Phi(x, z ; t) & =\left(c+b_{0}\right)\left(x-x_{0}-c t\right) \\
& +\sum_{j=1}^{N} b_{j} \frac{\cosh j k(z+h)}{\cosh j k h} \sin j k\left(x-x_{0}-c t\right) \\
& -\left[g(R-h)-\frac{1}{2} c^{2}\right] t \\
\eta(x ; t)=\frac{1}{2} a_{0} & +\sum_{j=1}^{N} a_{j} \cos j k\left(x-x_{0}-c t\right)
\end{aligned}
$$

where $c$ is the wave velocity, $x_{0}$ is the initial phase shift, $k$ is the wave number and $R$ is a constant. The coefficients $a_{j}$ and $b_{j}$ are found by substituting these series into the nonlinear free surface conditions (3-4) and then solving the resulting (nonlinear) system of equations with Newton's iteration method.

In the numerical calculations we necessarily work on a space interval of finite length. Therefore, we approximate the solitary waves by their periodic equivalents, the cnoidal waves, of long wavelength $\ell$, say $\ell \gg h$ and $\ell \gg a$. Our strategy is to obtain a numerical approximation to a solitary wave by taking the wavelength $\ell=2 \pi / k$ in $(20-21)$ extremely large with respect to the wave height and the water depth, such that the wave crests are confined to a relatively small region, where the wave troughs are very long. To illustrate this process we plotted in Fig. 2 three waves of amplitude $a=0.1$ on water depth $h=0.5$; the wavelengths are $\ell=5,10,20$. It can be seen that the waves converge rapidly to a uniform shape; since the wave troughs become more flat and wider, the wave crests are more and more separated and can therefore be seen as "solitary" waves.

In order to compute the wave propagation for a longer time than the (finite) wave period, we artificially create a continuation of the troughs on both sides: 


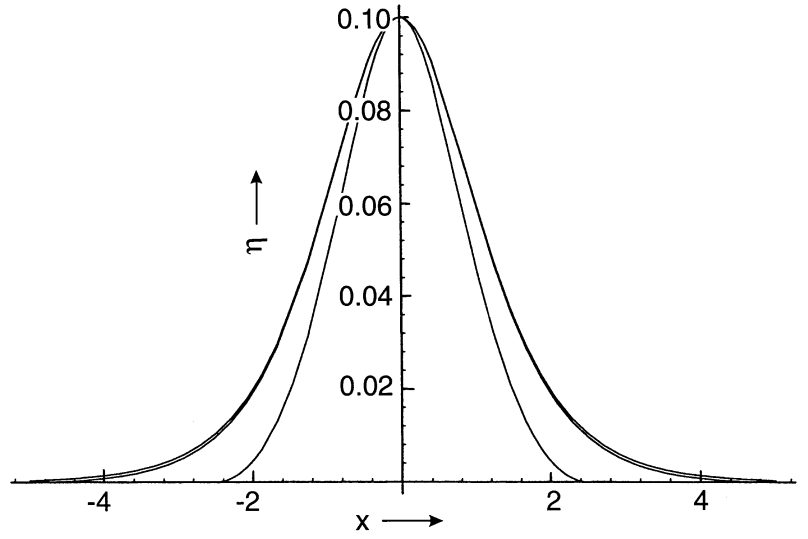

Fig. 2. Solitary wave approximation using steady, periodic waves with small amplitude-wavelength and depth-wavelength ratio

$$
\begin{aligned}
\left|x-x_{0}\right| \leq \frac{\ell}{2}: \Phi \text { and } \eta \quad \text { as given above } & \\
\left|x-x_{0}\right|>\frac{\ell}{2}: \Phi(x, z=\eta(x))= & \Phi\left(x_{1}\right) \\
& +\Phi_{x}\left(x_{1}\right)\left(x-x_{1}\right) \\
\eta(x)=\eta\left(x_{1}\right), \quad \text { where } \quad x_{1}= & x_{0}+\frac{\ell}{2} .
\end{aligned}
$$

The scheme proposed by Rienecker and Fenton (1981) (abbreviated as RF-scheme) calculates the Fourier coefficients of a cnoidal wave at given depth $h$ with (cresttrough) amplitude $a$, denoted as $\mathscr{C}(h, a)$. When the amplitude is sufficiently large, $\mathscr{C}(h, a)$ resembles a solitary wave above a layer of depth somewhat smaller than $h$. Recall that a solitary wave is a wave of elevation, different from $\mathscr{C}(h, a)$ which is constructed to have zero mass. This effect of depth change has to be inverted when we look for solitary waves above a layer of water of given depth. In order to find a wave (with its crest at $x=0$ ) with amplitude $a$ above a layer of depth $h$, we use the above RFscheme in the following iteration process:

1. RF-scheme: calculate the Fourier coefficients of $\mathscr{C}\left(h_{n}, a\right)$, the trough depth is $\delta_{n}=-\eta_{\mathscr{C}\left(h_{n}, a\right)}(x=\ell / 2)$,

2. take $h_{n+1}=h_{n}+\delta_{n}$

and we start with $h_{0}=h$. We carry out the iteration process above until $\left|h_{n}-\delta_{n}-h\right|<\beta$, with $\beta$ positive and very small. The RF-scheme also computes the Fourier coefficients of the corresponding velocity potential and the wave velocity $\lambda_{e}$. The procedure above leads to a cnoidal wave $\mathscr{C}\left(h_{n}, a\right)$, which is a wave of amplitude $a$ above a layer of depth $h_{n}-\delta_{n} \approx h$, so it resembles a solitary wave of amplitude $a$ above a layer of depth $h$. Since it is obtained from the exact equations, we call it the exact solitary wave and denote it from now on as $S_{e}$.

\section{2}

Solitary waves $S_{a}$ for the KdV-model

The solitary waves for the KdV-model above an even bottom are the well-known KdV-solitary waves. We will denote these by $S_{a}(h, \gamma)$; they are expressed in the physical variable $\gamma$, where $\gamma$ is the horizontal momentum; $\gamma$ is di- rectly related (in a one-to-one sense) to the amplitude $a$, see Eq. (24) below.

The solitary wave $S_{a}(h, \gamma)$ with velocity $\lambda$ can be written explicitly as

$S_{a}(h, \gamma)(x, t)=a \operatorname{sech}^{2} b(x-\lambda t)$,

with

$a=3 g\left(\frac{\gamma}{4}\right)^{2 / 3} c^{-4 / 3}, \quad b=\frac{3}{2} g^{2}\left(\frac{\gamma}{4}\right)^{1 / 3} c^{-11 / 3}$

and the value of the corresponding Hamiltonian is

$H\left(S_{a}\right)=c \gamma+\frac{18}{5} \epsilon g^{2}\left(\frac{\gamma}{4}\right)^{5 / 3} c^{-7 / 3}$.

Note that in (25) the potential energy contributes a factor $\frac{1}{2} c \gamma$; the rest is from the approximate kinetic energy. The velocity can be calculated directly from the Hamiltonian:

$\lambda=\frac{\mathrm{d}}{\mathrm{d} \gamma} H\left(S_{a}\right)=c+\frac{3}{2} \epsilon g^{2}\left(\frac{\gamma}{4}\right)^{2 / 3} c^{-7 / 3}$.

\section{3}

Comparison of $\mathrm{S}_{\mathrm{e}}$ and $\mathrm{S}_{\mathrm{a}}$

When comparing the solitary waves of the two models, an essential difficulty arises since the $\mathrm{KdV}$-model contains a parameter $\epsilon$ that was introduced to invoke the Boussinesq approximation. We will show that the choice of $\epsilon=0.7$ will lead to results that are comparable over a long range of wave-amplitudes (longer than will arise in the soliton runup process).

First we investigate the wave shapes. Above a flat bottom with $h=0.50$, waves $S_{e}$ and $S_{a}$ are compared for various amplitudes: $a=0.05,0.10, \ldots, 0.30$. Figure 3 shows that the shapes of $S_{e}$ and $S_{a}$ are nicely comparable, especially for waves with small amplitudes.

Next, we compare their mass, amplitude, energy and velocity. Evaluating $M$ for $S_{a}$ leads to the following explicit expression:

$M\left(S_{a}\right)=\frac{4}{g}\left(\frac{\gamma}{4}\right)^{1 / 3} c^{11 / 6}$.

The total energy and the momentum are given by (25) and (24) respectively. For $S_{e}$, calculating $M, I$ and $H$ consists of integrating harmonic functions over one period, that is over their wavelength $\ell$.

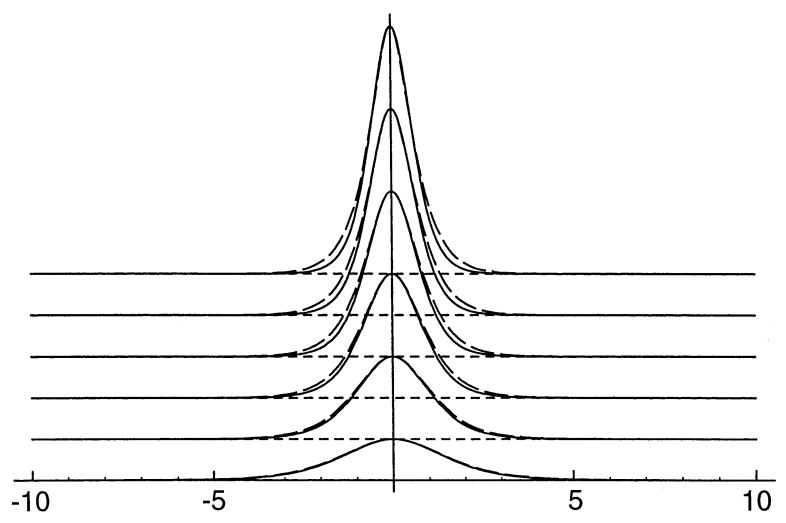

Fig. 3. Solitary waves $S_{a}$ for $\epsilon=0.7$ (solid lines) and $S_{e}$ (dashed lines) with amplitude $a=0.05,0.10, \ldots, 0.30$ 

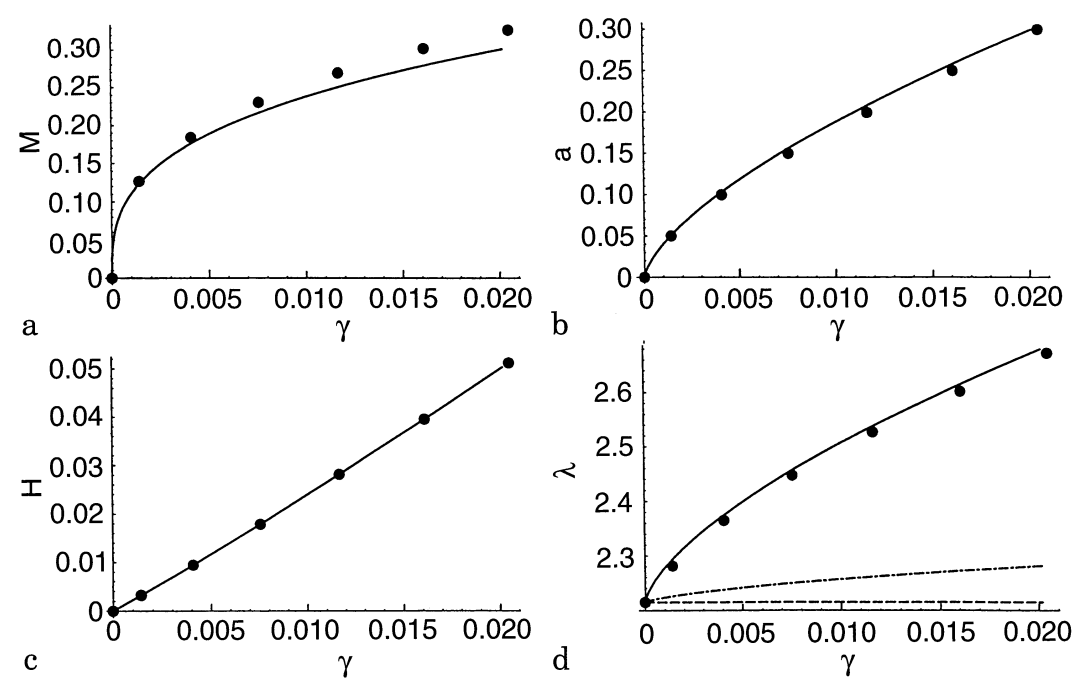

Fig. 4a-d. The value of mass (a), amplitude (b), energy (c) and velocity (d) versus momentum for $S_{a}$ (solid lines) and $S_{e}$ (dots) for $h=0.5$. The velocity $\lambda$ is plotted with three choices of $\epsilon$ : - - - for $\epsilon=0,-\cdot-\cdot-$. for $\epsilon=0.1$ and - for $\epsilon=0.7$

The results are plotted in Fig. $4 \mathrm{a}, \mathrm{b}$ and $\mathrm{c}$. In those three figures, the dots of $S_{e}$ are very close to the lines of $S_{a}$. This means that waves $S_{e}$ and $S_{a}$ of the same amplitude have nearly the same value of mass, energy and momentum. Quantities like amplitude, mass, momentum (and energy) can be viewed as various norms in the function space, that is the maximum norm, the $L^{1}$-norm and the $L^{2}$-norm respectively. Hence, Figs. $4 a, b$, and $c$ show the differences between $S_{a}$ and $S_{e}$ with respect to those norms. The energy $H\left(S_{a}\right)$ as given in (25) is plotted using $\epsilon=0.7$. This value of $\epsilon$ is motivated from Fig. $4 \mathrm{~d}$, in which the velocity $\lambda$ is plotted with several choices of $\epsilon$. For $\epsilon=0.7$, the line $\lambda$ matches the dots of $\lambda_{e}$; this means that for $\epsilon=0.7$ the $\mathrm{KdV}$-top model and the exact equations are comparable over a long range of wave amplitudes. This specific value of $\epsilon$ will also be used later in Section 6, to describe the splitting process analytically.

\section{4}

\section{BEM calculations of splitting}

In this section we describe the numerical aspects of the BEM-calculations, both the principles of the algorithm and a discussion of the computer performance in the specific calculations of the solitary wave splitting.

\section{1}

Outline of the numerical method

Since the time-dependence comes into the water wave problem through the free surface conditions (3-4) only, the exact problem can be split into two subproblems which are solved step by step.

The time-independent part is governed by Laplace's Eq. (1) which, using Green's identity, is transformed into a boundary integral equation (BIE):

$$
\begin{aligned}
\frac{1}{2} \phi(\vec{x})= & \int_{\partial \Omega}\left[\frac{\partial \phi}{\partial n_{\xi}}(\vec{\xi}) G(|\vec{x}-\vec{\xi}|)\right. \\
& \left.-\phi(\vec{\xi}) \frac{\partial G}{\partial n_{\xi}}(|\vec{x}-\vec{\xi}|)\right] \mathrm{d} S_{\xi},
\end{aligned}
$$

where $G$ is the Green's function $\left(G(r)=\frac{1}{2 \pi} \ln \frac{1}{r}\right.$ in $\left.2 \mathrm{D}\right)$ and integration is over the boundary of the fluid domain $\Omega$. In this continuous form, the BIE is applied in each point $\vec{x}$ on $\partial \Omega$. In a discretized form the boundary is approximated by a finite number of boundary elements, each represented (in our approach) by one collocation point $\vec{x}_{i}$ situated in the middle of the element. The BIE is applied in each point $\vec{x}_{i}$, so that a system of linear equations is obtained:

$\frac{1}{2} \phi^{i}=\sum_{j=1}^{N}\left[C_{s}^{i j} \phi_{n}^{j}+C_{d}^{i j} \phi^{j}\right]$,

where $C_{s}^{i j}$ and $C_{d}^{i j}$ are the source and dipole coefficients respectively and summation is over all $N$ collocation points. Substitution of $\phi$ for Dirichlet boundaries and $\phi_{n}$ for Neumann boundaries yields $N$ linear equations in exactly $N$ unknowns, which can be solved using direct methods (e.g. Gaussian elimination) or iterative methods (e.g. conjugate gradients type methods). The solution yields $\phi_{n}$ for the Dirichlet boundaries and $\phi$ for the Neumann boundaries.

Next, we have to solve the time dependent part of the problem, especially for the evolution of the free surface. The new positions of the collocation points are determined by integrating the kinematic conditions, including (3), in time. The new values of the potential at the free surface collocation points are obtained by integrating the dynamic condition (4). For the time marching we use a fourth order classical Runge-Kutta scheme, which implies that the BVP has to be solved on four levels for each time step. For a detailed description of the mathematical model and the computational method we refer to van Daalen (1993).

\section{2}

Numerical verification of soliton propagation

To demonstrate the accuracy and stability of the numerical method, we simulated a wave with amplitude $a=0.10$ and wavelength $\ell=40$ on $h=0.50$ water depth. The Fourier series for the potential and the elevation are computed up to 100 terms each. The wave troughs were artificially elongated as described by Eq. (22); the domain length is $L=2.5 \ell=100$.

Figure 5 shows the wave at subsequent stages of evolution, for $t=0,5, \ldots, 40$. Figures $6 \mathrm{a}, \mathrm{b}$ and $\mathrm{c}$ show the 


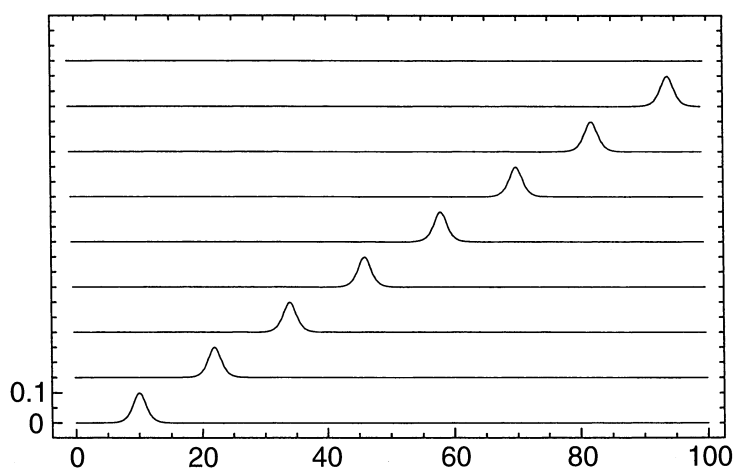

Fig. 5. Solitary wave propagating over a flat bottom ("waterfall view")

mass, energy and the absolute maximum error in the elevation versus time. Clearly, mass and energy are conserved (as they should be); note that the results are shown on a relatively detailed scale. The maximum error in the elevation is, although growing, less than $1.5 \%$ of the wave amplitude at the end of the simulation.

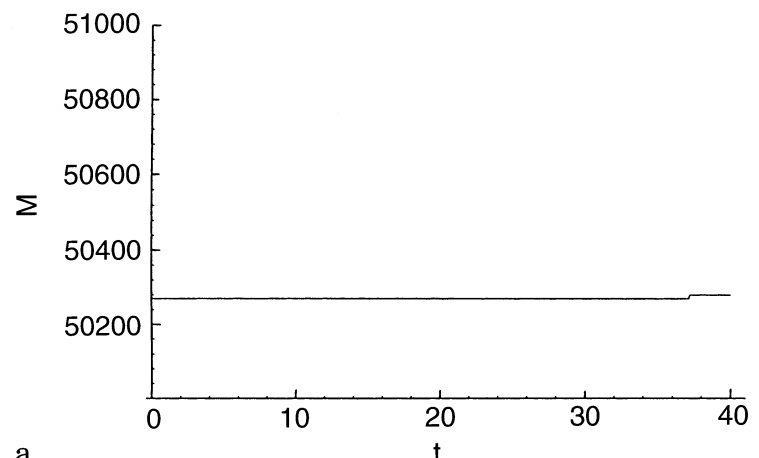

a
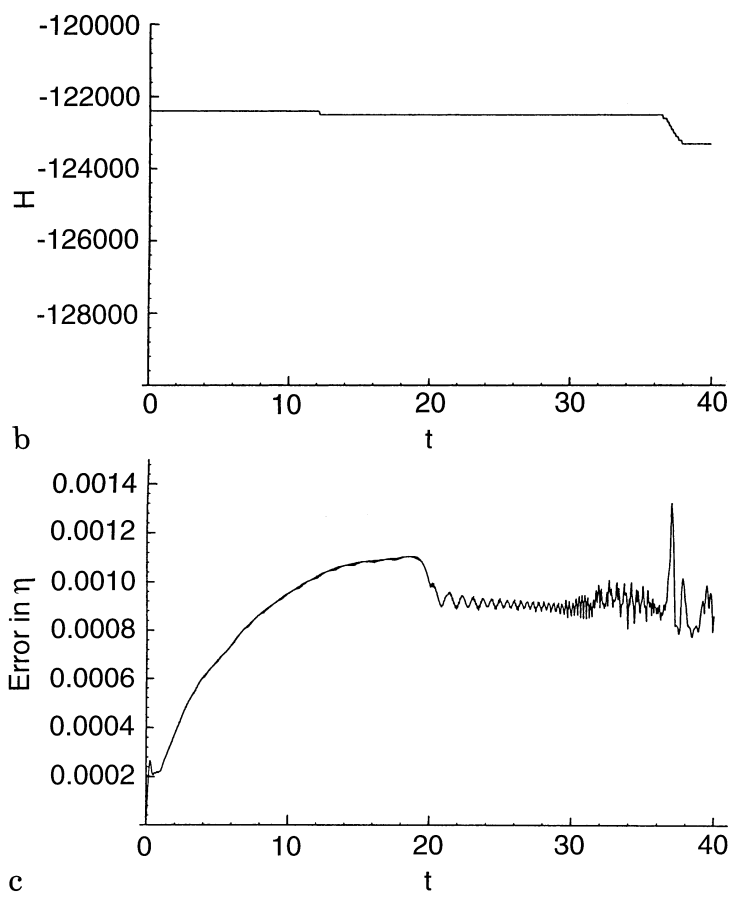

Fig. 6a-c. Mass (a), energy (b) and maximum absolute error in elevation (c) versus time for a solitary wave propagating over a flat bottom
4.3

Computational aspects

Here we present some information concerning the computational aspects of the numerical calculations.

The BEM-computations were done on a CRAY-YMP C90 supercomputer with a clock speed of 4167 Picoseconds; in all computations we used one processor.

The number of panels used is 400 on the free surface, 10 on the inflow and outflow boundaries and 400 on the bottom, that is 820 in total. A fine grid was necessary on the bottom too, because large panels would induce nonphysical waves at the free surface; as a rule of thumb, the panel size should be smaller than the water depth. The time step was $\Delta t=0.01$, so that 4000 time steps were needed to simulate a wave during 40 seconds.

Each computation consumed about 22,000 CPU seconds (approximately $6 \mathrm{CPU}$ hours), where the larger part (about $88 \%$ ) was on the account of the analytical calculation of the influence coefficients (4000 steps $\times 4$ time levels $\times 820^{2}$ coefficients $\approx 10^{10}$ calculations involving the expensive arctan operation). The solution of the linear system of equations took approximately $10 \%$ of the CPU-time. The remaining costs were due to time stepping and I/O. The maximum memory used was 1.73 MWords; on average a computation involved 2.75 MWords of data transfer; the overall performance was 280 Mflops.

5

Analytic description of splitting

In this section we present an approximate analytic description of the splitting process using the KdV-top model. It is shown that conservation of mass and energy alone suffice to obtain a quasi-homogeneous approximation.

5.1 Quasi-homogeneous approximation

The idea of the quasi-homogeneous approximation is simple once the basic evolution pattern is accepted.

In view of the limited change in depth that we consider, it can be expected - and argued, see van Groesen (1996) and Pudjaprasetya and van Groesen (1997) - that the single soliton during run-up will distort into two solitontype of waves plus some additional tail. Neglecting the tail for the moment, the simplest approximation is to assume that during the splitting process the wave pattern will consist of a superposition of two single solitons, each one determined by its value of momentum. An additional simplification arises if we assume that both solitons are at the same depth, the value of the depth changing according to the position of the combined waves. So, neglecting for a moment the precise positions of the waves (that can be calculated a posteriori), we assume that the elevation evolves (parameterized by $h$ ) as

$U_{a}=S_{a}\left(h, \gamma_{+}\right)+S_{a}\left(h, \gamma_{-}\right)$,

where $\gamma_{+}, \gamma_{-}$are the changing momenta of the constituent waves. The determination of their dependence on $h$ will be sufficient for the complete description of this quasi-

homogeneous approximation. 
5.2

Mass and energy conservation

Starting with a single soliton of momentum $\gamma_{0}$ above a flat bottom at depth $h_{0}$, for which we take $\gamma_{+}=\gamma_{0}$ and $\gamma_{-}=0$, the evolution

$h \mapsto\left(\gamma_{+}(h), \gamma_{-}(h)\right)$

will be determined from mass and energy conservation since these quantities are conserved for any exact solution of KdV-top:

$\left\{\begin{array}{c}H\left(S_{a}\left(h, \gamma_{+}(h)\right)\right)+H\left(S_{a}\left(h, \gamma_{-}(h)\right)\right)=H\left(S_{a}\left(h_{0}, \gamma_{0}\right)\right) \\ M\left(S_{a}\left(h, \gamma_{+}(h)\right)\right)+M\left(S_{a}\left(h, \gamma_{-}(h)\right)\right)=M\left(S_{a}\left(h_{0}, \gamma_{0}\right)\right) .\end{array}\right.$

Substitution of the explicit expressions (27) and (25) into (31) leads to two equations from which the dependence can be calculated. Results will be shown in the next section.

\section{6}

Comparison of numerical and analytic splitting

In this section we present the results of the BEM-calculations and the analytic quasi-homogeneous description of the splitting process and compare the results. For every time step, the numerical elevation is denoted as $U_{e}$, to be compared with the analytical elevation $U_{a}$.

\section{1}

\section{BEM soliton run-up}

The initial conditions at the free surface are given by (20-21), with wave amplitude $a_{0}=0.10$ and water depth $h_{0}=0.50$. The length of the numerical wave tank is $L=100$ and we used the above described method to extend the wave, following (22), thus creating an initial solitary wave.

Three cases for soliton run-up were simulated: in all cases, the bottom variation is between $x=15$ and $x=22.5$, with the depth decreasing from $h_{0}=0.50$ to $h_{1}=0.40$ (Case 1, slope 1:75), $h_{1}=0.35$ (Case 2, slope $1: 50$ ), and $h_{1}=0.30$ (Case 3, slope 1:37.5). After $x=22.5$, in the shallower region, the bottom is horizontal again.

The results are shown in Figs. 7, 8 and 9. The waves are shown at the subsequent stages of evolution

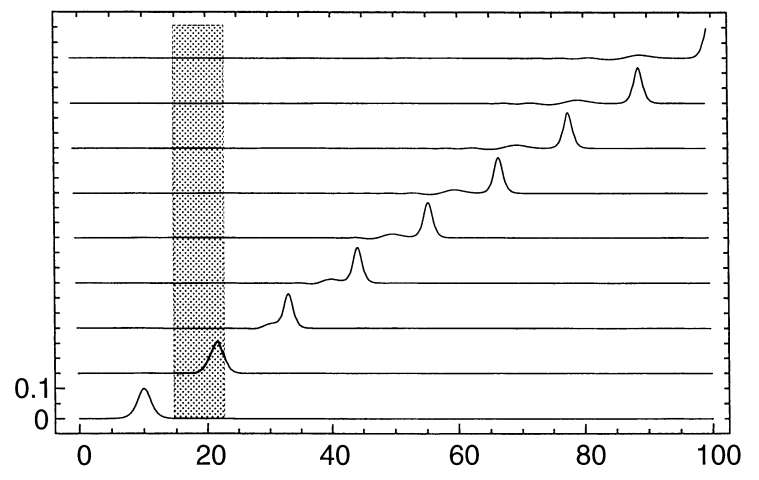

Fig. 7. Solitary wave propagating over a sloping bottom (waterfall view). Wave profiles are shown at $t=0,5, \ldots, 40$. Case 1: $h_{0}=0.50 \downarrow h_{1}=0.40$ (1:75 slope)

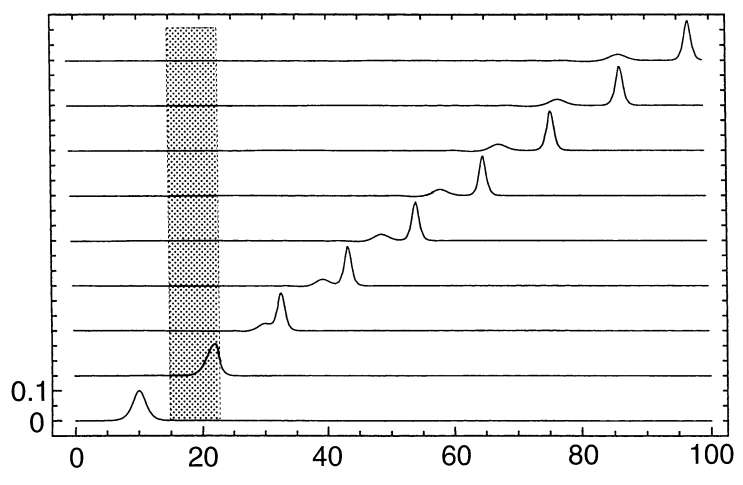

Fig. 8. Solitary wave propagating over a sloping bottom. Wave

(1:50 slope)

$t=0,5, \ldots, 40$. From these plots it becomes clear that the effect of decreasing depth on the wave is fourfold: the wave becomes higher and steeper, its speed decreases and it splits into a 2-soliton with an additional tail.

\section{2}

Analytical soliton run-up

The initial wave is a solitary wave with amplitude

$a_{0}=0.10$ running up a bottom topography with decreasing depth from $h_{0}=0.50$ to $h_{1}=0.30$ (with slope 1:37.5), corresponding to Case 3 of the BEM-computations.

Figure 10 shows the two-soliton $U_{a}$ with $\gamma_{+}(h)$ and $\gamma_{-}(h)$ obtained from (31), at subsequent times $t=0,5, \ldots, 40$.

The second wave $S_{a}\left(h, \gamma_{-}\right)$starts to split off from the first wave $S_{a}\left(h, \gamma_{+}\right)$directly after the depth decrease. The first soliton runs faster than the second one, they both are travelling with velocities larger than the linear velocity $c_{0}$.

In the result of the BEM-calculation, $U_{e}$ in Fig. 9, besides the two-soliton shape, we get an additional tail. In the analytical approach, the tail is neglected since we use $U_{a}$ as the base function. Apart from the tail, the two-soliton shape of $U_{e}$ is qualitatively comparable with $U_{a}$, a quantitative comparison is given in $\S 6.3$.

We observe that in both the numerical case and the analytical case the splitting process takes quite some time and when the solitons are separated, they are already far enough from the unevenness. The time needed to complete the splitting process is directly related to the velocity dif-

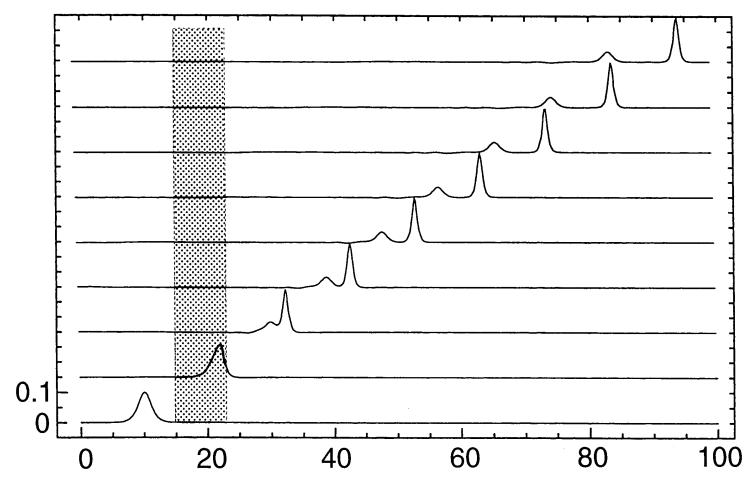

Fig. 9. Solitary wave propagating over a sloping bottom. Wave profiles are shown at $t=0,5, \ldots, 40$. Case $3: h_{0}=0.50 \downarrow h_{1}=0.30$ (1:37.5 slope) 


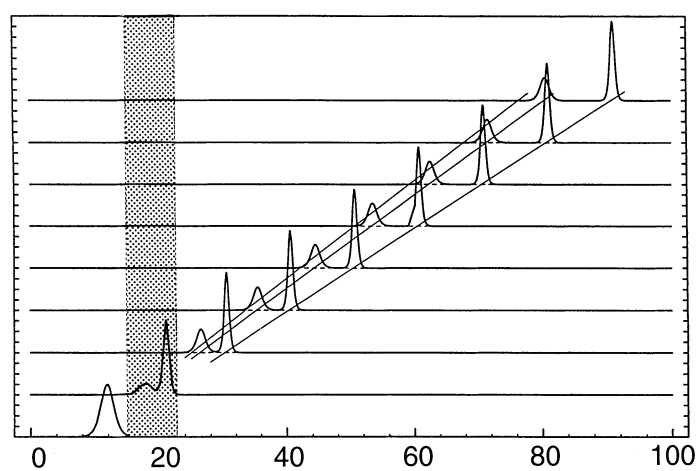

Fig. 10. Analytic approximation of soliton splitting during run up. The grey strip is a region of decreasing depth $\left(h_{0}=0.50 \downarrow h_{1}=0.30\right)$ with uniform-slope

ferences between the two solitons; we discuss this in the next paragraph.

\section{3}

Comparison of evolving observables

Using $a_{0}$ and $h_{0}$ and from (24) the initial momentum $\gamma_{0}$, Eqs. (31) determine the evolutions of $\gamma_{+}, \gamma_{-}$with respect to the changing depth $h$. The solvability of (31) gives a lower bound for $h$, say $h_{L}$. When we plot $\gamma_{+}(h)$ and $\gamma_{-}(h)$ for $h \in\left[h_{L}, h_{0}\right]$ we get a trajectory of a soliton running up, the full line in Fig. 11a. Initially, we have $\gamma_{+}\left(h_{0}\right)=\gamma_{0}$ and $\gamma_{-}\left(h_{0}\right)=0$, then both increase with decreasing depth, but after a certain depth, close to $h_{L}, \gamma_{+}$starts to decrease.

Next, we substitute the momenta $\gamma_{+}(h)$ and $\gamma_{-}(h)$ in the expressions for the corresponding amplitudes and velocities; the results are plotted in Figs. $11 \mathrm{~b}$ and c. The velocity difference

$\lambda_{+}-\lambda_{-}=\frac{3}{2} \epsilon g^{2} c^{-7 / 3}\left(\left(\frac{\gamma_{+}}{4}\right)^{2 / 3}-\left(\frac{\gamma_{-}}{4}\right)^{2 / 3}\right)$

depends on the choice of $\epsilon$. Here, we choose $\epsilon=0.7$, since for this value the results of the KdV-top model and the exact equations are comparable.

The amplitudes of the two waves of soliton shapes (the dots in Fig. 11b) are measured from Figs. 7-9, after they have separated completely. Note that for $h \downarrow 0.40$, the second wave is very low, so it is not measurable. The dots in Fig. 11a are the corresponding momenta of $S_{a}$ (and not $S_{e}$, for the sake of simplicity) of the measured amplitudes above. The dots in Fig. 11c represent the velocities of the numerical waves, measured from Figs. 7-9.

The dashed lines in Figs. 11a, b, c are data of splitted soliton obtained from the Inverse Scattering Theory (IST) by Johnson (1973). He uses the IST for KdV flat bottom to explain the same problem. Using this approach the splitting process starts only after the wave has entered the flat bottom region.

\section{7}

\section{Conclusions}

In this paper we have presented a comparative study of numerical and analytical calculations for solitary waves splitting due to varying bottom topography. The numerical results were obtained for the full set of (exact) equa-
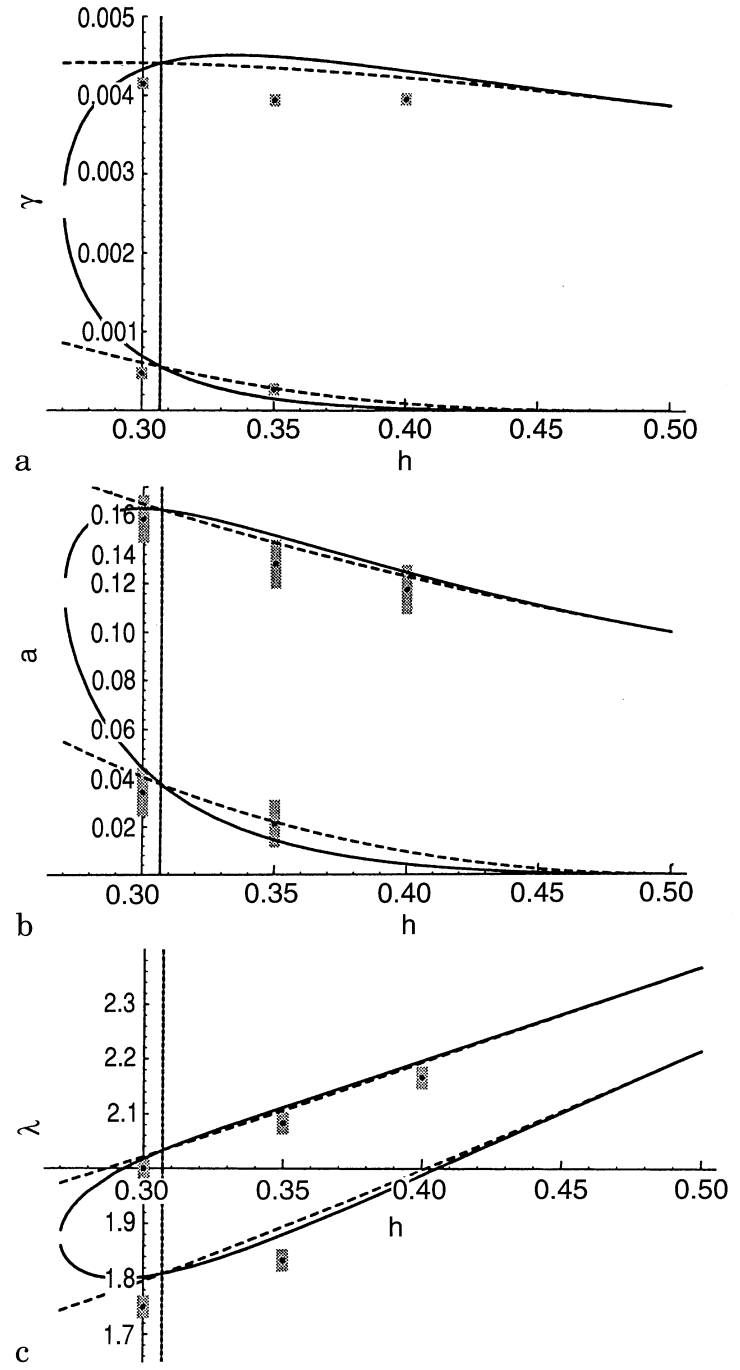

Fig. 11a-c. Soliton data after splitting: analytical results (solid lines), BEM-calculations (dots) and IST-results (dashes). The error bars around the dots show the possible deviations caused by measurement-errors. The vertical lines $h=\left(\frac{1}{3}\right)^{4 / 9} h_{0}\left(>h_{L}\right)$ (predicted from IST, by Johnson (1973)) indicate the eigen-depth for the pure twosoliton

tions for the classical water wave problem; an accurate time domain boundary element method was used. The analytical predictions were generated with a $\mathrm{KdV}$-equation modified for slowly varying water depth.

Detailed information was presented about the numerical approximation of solitary waves, using the nonlinear steady wave solution of Rienecker and Fenton (1981). It was demonstrated in advance, by checking the undisturbed (flat bottom) case first, that the numerical computations are sufficiently accurate to study the detailed process of splitting.

The comparison was made both from a qualitative (phenomenological) point of view and in a quantitative respect, evaluating several wave parameters (mass, energy, etc.) for the numerical and analytical solutions.

The numerical results seem to confirm the analytical predictions; the effect of decreasing depth on a single soliton is fourfold; the wave becomes higher and steeper, its speed decreases and its splits (roughly) into a 2-soliton 
with an additional tail (which is absent in the analytical model). These effects are more clearly present for stronger variations in the water depth, as can be observed from Figs. 7-9.

In a quantitative sense, the numerical results are in good agreement with the analytical values that can be derived for the momentum, amplitude and velocity as functions of depth. Although there are systematic overpredictions and underpredictions, the numerical results support the validity of the assumptions underlying the analytical $\mathrm{KdV}$ model.

More detailed numerical computations and, perhaps, a more refined analytical model will shed some light on problems concerning the number of solitons generated, the characteristics of the tail and other questions that undoubtedly will arise in future research.

\section{References}

Grimshaw, R. (1970): The solitary wave in water of variable depth. J. Fluid Mech. 42: 639-656

Grimshaw, R. (1971): The solitary wave in water of variable depth. Part 2. J. Fluid Mech. 46: 611-622

Johnson, R. S. (1972): Some numerical solutions of a variable-coefficient Korteweg-de Vries equation (with application to solitary wave development on a shelf). J. Fluid Mech. 54: 81-91

Johnson, R. S. (1973): On the development of a solitary wave moving over an uneven bottom. Proc. Cambr. Phil. Soc. 73: 183-203

Johnson, R. S. (1994): Solitary wave, soliton and shelf evolution over variable depth. J. Fluid Mech. 276: 125-138
Karpman, V. I.; Maslov, E. M. (1978): Structure of tails produced under the action of perturbation on solitons. Sov. Phys. JETP 48: 252259

Knickerbocker, C. J.; Newell, A.C. (1980): Shelves and the Kortewegde Vries equation. J. Fluid Mech. 98: 803-818

Newell, A.C. (1985): Soliton in mathematics and physics. Society for Industrial and Applied Mathematics, Philadelphia, Pennsylvania

Pudjaprasetya, S. R.; van Groesen, E. (1997): Uni-directional waves over slowly varying bottom. Part III (in preparation)

Rienecker, M. M.; Fenton, J. D. (1981): A Fourier approximation method for steady water waves. J. Fluid Mech. 104: 119-137

van Daalen, E. F. G. (1993): Numerical and Theoretical Studies of

Water Waves and Floating Bodies. PhD-thesis, University of Twente, Enschede, The Netherlands

van Daalen, E. F. G. (1995): Solitary wave split-up due to a sloping bottom. In: Computational Mechanics '95, Theory and Applications, pp. 2927-2932. (Eds: S. N. Atluri, G. Yagawa, T. A. Cruse.) (Proceedings of the International Conference on Computational Engineering Science, Hawaii, USA, July 1995)

van Groesen, E.; de Jager, E. M. (1994): Mathematical Structures in Continuous Dynamical Systems. In: Studies in Mathematical Physics. Amsterdam: North-Holland, Elsevier van Groesen, E. (1996): A phenomenological description of soliton splitting during run up. To appear in the Proceedings of the Workshop on Problems in the Theory of Nonlinear Hydrodynamic Waves, Luminy, France, May 1995

van Groesen, E.; Pudjaprasetya, S. R. (1993): Uni-directional waves over slowly varying bottom. Part I: Derivation of a KdV-type of equation. Wave Motion 18: 345-370 